\title{
On the quasi-steady-state assumption applied to Michaelis-Menten and suicide substrate reactions with diffusion
}

\author{
By P. K. Maini ${ }^{1}, \mathrm{Meghan}_{\text {A. BuRKe }}{ }^{1}$ and J. D. MuRRaY ${ }^{2}$ \\ ${ }^{1}$ Centre for Mathematical Biology, Mathematical Institute, 24-29 St Giles', \\ Oxford OX1 3LB, U.K. \\ ${ }^{2}$ Applied Mathematics Department FS-20, University of Washington, Seattle, \\ Washington 98195, U.S.A.
}

\begin{abstract}
We consider a recent extension to the validity of the quasi-steady-state assumption (QSSA) which includes the case where the ratio of the initial enzyme to substrate concentration is not necessarily small. We extend the analysis to include diffusion of substrate, in which case the initial enzyme to substrate ratio is spatially dependent and no longer constant. We show that the region in which the QSSA holds depends on the nature of the enzyme-substrate reaction: if the enzyme is inactivated by the substrate then the QSSA holds in a growing disc; if the enzyme is unchanged after reaction then the QSSA holds in a ring travelling through space.
\end{abstract}

\section{Introduction}

Many biochemical reactions exhibit an initial fast transient followed by a slowly varying change in reactant concentration. The system of ordinary differential equations describing such reactions are usually solved analytically using the pseudosteady-state hypothesis (PSSH) or the quasi-steady-state assumption (QSSA). Essential to this technique is the isolation of a small parameter to enable a singular perturbation procedure to be employed. It is customary to take this small parameter to be the ratio of initial enzyme to substrate concentration (see Michaelis \& Menten 1913; Murray 1989; Laidler \& Bunting 1973). However, in many reactions of biochemical interest this ratio may not be small (Sols \& Marcos 1970) nor even constant. Thus the standard analysis is inadequate for a wide range of biochemically relevant problems.

Segel \& Slemrod (1989) re-examined the Michaelis-Menten reaction and introduced a formulation of the problem containing a new, more general, small parameter. Their parameter not only includes the usual one as a special case, but also allows for the possibility of a singular perturbation analysis for certain problems in which the initial enzyme to substrate concentration is not small. This analysis was applied to a more complicated reaction scheme by Frenzen \& Maini (1988).

Burke et al. (1990) extended the analysis to the case where some of the enzyme is rendered inactive by reaction with the substrate. They specifically considered the mechanism-based inhibitor, or 'suicide substrate' system, represented by (Walsh et al. 1978) 


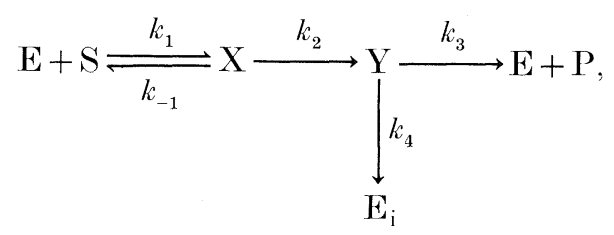

where $\mathrm{E}, \mathrm{S}$ and $\mathrm{P}$ are enzyme, substrate and product respectively; $\mathrm{X}$ and $\mathrm{Y}$ are enzyme-substrate intermediates; $\mathrm{E}_{\mathrm{i}}$ is inactivated enzyme, and the $k$ s are positive rate constants. In this system the enzyme converts the substrate into an inhibitor which, in turn, can irreversibly inactivate the enzyme. The ratio $k_{3} / k_{4}$ is called the partition ratio, and is a measure of the fraction of enzyme inactivated. The substrate $\mathrm{S}$ is called a suicide substrate.

This class of reaction is of considerable experimental interest (Rando 1977) because a suicide substrate provides a means to inactivate a specific enzyme. For example, suicide substrates have been investigated for use in the treatment of depression and certain types of tumours (monoamine oxidase and ornithine decarboxylase inhibitors respectively (Seiler et al. 1978)) and in epilepsy (brain GABA transaminase inhibitors (Walsh 1984)). In such applications it is important to find the conditions that determine whether the substrate is exhausted before the inactivation of all the enzyme. This has been analysed by Waley (1980) and Tatsunami et al. (1981). Both of these studies used approximations based on a form of QSSA which assumes that $E_{0} / S_{0}$ is small, thus their results are inadequate in the limit when $E_{0} / S_{0}$ approaches 1 . Burke et al. (1990) used the more general form of the small parameter to obtain an analytic, uniformly valid solution for all time which encompasses this case. Their solution clearly shows the transition from the fasttransient, or pre-steady-state, phase to the quasi-steady-state phase. It also provides quantitative expressions for the time evolution of the reactants in terms of the rate constants.

In this paper we extend the analysis to the more biochemically realistic case in which the substrate is allowed to diffuse over the region containing the immobilized enzyme. In $\S 2$ we derive the model equations for this system using the law of mass action. For completeness, in $\S 3$ we briefly summarize the results for the case where there is no diffusion (see Burke et al. (1990) for full details). When substrate diffusion is included, we show (\$4) that the region wherein the QsSA holds depends on space and time in a manner that varies considerably depending upon the type of reaction (inactivation or non-inactivation of enzyme) in question. We focus our attention on the aforementioned enzyme-suicide-substrate reaction and the Michaelis-Menten reaction.

\section{Model equations}

By using the law of mass action, the rate equations for reaction (1), where the substrate alone can diffuse, are given by

$$
\begin{aligned}
\partial S / \partial t & =-k_{1} E S+k_{-1} X+D \nabla^{2} S \\
\partial E / \partial t & =-k_{1} E S+k_{-1} X+k_{3} Y \\
\partial X / \partial t & =k_{1} E S-k_{-1} X-k_{2} X \\
\partial Y / \partial t & =k_{2} X-k_{3} Y-k_{4} Y \\
\partial E_{\mathrm{i}} / \partial t & =k_{4} Y \\
\partial P / \partial t & =k_{3} Y
\end{aligned}
$$

Phil. Trans. R. Soc. Lond. A (1991) 
where, for notational convenience, we now represent the concentration of each reactant by the letters specified in (1). Note that the concentrations are functions of both the spatial coordinate, $\boldsymbol{r}$, and time, $t$. We have modelled substrate diffusion using Fick's Law (see, for example, Murray 1989) where we have assumed that $D$, the diffusion coefficient, is a positive constant. Typical experimental initial conditions are

$$
E(\boldsymbol{r}, 0)=E_{0}, S(\boldsymbol{r}, 0)=S_{0}(\boldsymbol{r}), X(\boldsymbol{r}, 0)=Y(\boldsymbol{r}, 0)=E_{\mathbf{i}}(\boldsymbol{r}, 0)=P(\boldsymbol{r}, 0)=0,
$$

where $r \in \Omega$, the spatial domain, $E_{0}$ is the spatially independent initial enzyme concentration and $S_{0}(\boldsymbol{r})$ is some initial spatial distribution of substrate. To complete the mathematical formulation we assume that the domain boundaries are impermeable to substrate, hence we have zero flux conditions, $\nabla S=0$ on $\partial \Omega$, the boundary. As we will be considering a localized reaction on a very large domain the exact form of the boundary condition will have no effect during the time course of reaction considered.

We rewrite the system by noting the enzyme conservation relation (adding (3), (4), $(5)$, and $(6))$

$$
\begin{aligned}
\partial\left\{E+X+Y+E_{\mathrm{i}}\right\} / \partial t & =0 \\
\Rightarrow E+X+Y+E_{\mathrm{i}} & =E_{0} .
\end{aligned}
$$

By using (10) the original system of six equations becomes

$$
\begin{aligned}
\partial S / \partial t & =-k_{1}\left(E_{0}-X-Y-E_{\mathrm{i}}\right) S+k_{-1} X+D \nabla^{2} S \\
\partial X / \partial t & =k_{1}\left(E_{0}-X-Y-E_{\mathrm{i}}\right) S-\left(k_{-1}+k_{2}\right) X \\
\partial Y / \partial t & =k_{2} X-\left(k_{3}+k_{4}\right) Y \\
\partial E_{\mathrm{i}} / \partial t & =k_{4} Y \\
\partial P / \partial t & =k_{3} Y
\end{aligned}
$$

with the initial conditions

$$
S(\boldsymbol{r}, 0)=S_{0}(\boldsymbol{r}), X(\boldsymbol{r}, 0)=Y(\boldsymbol{r}, 0)=E_{\mathbf{i}}(\boldsymbol{r}, 0)=P(\boldsymbol{r}, 0)=0 .
$$

The equation for the product is decoupled from the other equations thus we need only solve (11)-(14) and substitute into (15) to find the product concentration.

\section{Previous results}

The special case of no diffusion, $D=0$, and $S_{0}(\boldsymbol{r})=S_{0}$, a constant, was studied in detail by Burke et al. (1990). In this case, the concentrations are functions only of time. Here we simply outline the main points of their analysis. The important features of the two distinct evolution phases is reflected in appropriate time scalings. In the fast-transient phase it is necessary to choose a time scaling such that the substrate concentration remains constant at leading order while the dimensionless time rate of change of the enzyme-substrate complexes and inactivated enzyme is non-zero. During the Qss phase it is necessary to choose a timescale on which the rate of change of the dimensionless substrate concentration is non-zero while the dimensionless derivatives of each of the other components should appear multiplied by a small parameter, to indicate that they are in steady state with respect to the instantaneous value of the substrate concentration. This is what is meant by Qss. By solving the resulting dimensionless system in each phase and using the method of 
matched asymptotic expansions, one can then obtain a solution uniformly valid in time.

The variables are non-dimensionalized by setting

$$
S=S_{0} s, \quad X=\frac{E_{0} S_{0}}{S_{0}+K} x, \quad Y=E_{0} y, \quad E_{\mathrm{i}}=E_{0} e_{\mathrm{i}}
$$

where

$$
K=\left(k_{-1}+k_{2}\right) / k_{1}
$$

and $s, x, y$ and $e_{\mathrm{i}}$ are now dimensionless variables. The dimensionless times are

$$
\tau=t / t_{\mathrm{p}}=t k_{1}\left(S_{0}+K\right)
$$

for the initial, fast-transient phase, and

$$
T=t / t_{\mathrm{s}}=t \epsilon\left(k_{-1}+k_{2}\right),
$$

where

$$
\epsilon=E_{0} /\left(S_{0}+K\right)
$$

for the Qss phase.

A further requirement is that the timescale of the fast-transient phase be much smaller than that of the Qss phase: that is, $t_{\mathrm{p}} / t_{\mathrm{s}} \ll 1$, which implies

$$
\epsilon /\left[1+\left(S_{0} / K\right)\right] \ll 1 .
$$

This clearly holds if $\epsilon \ll 1$. Hence the appropriate small parameter is $E_{0} /\left(S_{0}+K\right)$. This is confirmed by the subsequent singular perturbation analysis.

By using the scalings in (17) with (19) one obtains the non-dimensionalized version of equations (11)-(14) for the fast-transient phase. In the Qss phase it is assumed, as usual, that only the timescale need be changed and the appropriate non-dimensional rate equations are obtained using (20). This leads to the two sets of dimensionless rate equations for the two time periods. By using the technique of matched asymptotic expansions one can find uniformly valid solutions.

Burke et al. (1990) compared their analytic solutions with the numerical solutions of the full system and to previous approximations for several different sets of parameters. They found them to be more accurate for most of the time course of the reaction than previous approximations.

\section{Diffusing substrate}

The above analysis assumed that the concentration of the reactants varies only with time and is spatially independent. However, in many biologically relevant examples, the reactants diffuse so their concentration does depend on space. The corresponding model equations are then (11)-(14) with initial condition (16) and appropriate boundary conditions on $S$. We illustrate the analysis in this case by assuming that initially an amount $S_{0}$ of substrate is injected at the origin over a very short period of time, and take the initial condition for $S$ to be

$$
S(\boldsymbol{r}, 0)=S_{0} \delta(\boldsymbol{r})
$$

where $\delta(\boldsymbol{r})$ is the Dirac delta function. The spatial domain is taken to be the plane. By symmetry, for these initial conditions, the spatial distribution of each of the reactants is radially symmetric, and the appropriate boundary condition for $S$ at the 
origin is zero flux. At the edge of the domain we also assume zero flux boundary conditions for $S$, that is, we assume that the domain is impermeable to the substrate.

In this situation we cannot use the non-dimensionalization of $\S 3$ because the initial substrate concentration varies in space. However, we can use the expression for the small parameter $\epsilon$ to delimit regions in which the Qssa holds. We illustrate the procedure by comparing the results for the enzyme suicide substrate reaction with those for the Michaelis-Menten kinetics where the substrate can diffuse in both cases.

The Michaelis-Menten reaction has the form:

$$
\mathrm{E}+\mathrm{S} \underset{k_{-1}}{\stackrel{k_{1}}{\rightleftharpoons}} \mathrm{X} \stackrel{k_{2}}{\longrightarrow} \mathrm{E}+\mathrm{P},
$$

where E, S, X and P are enzyme, substrate, enzyme-substrate complex and product respectively, and the $k \mathrm{~s}$ are positive rate constants. The equations for this system, with diffusion of the substrate, are:

$$
\begin{aligned}
& \partial S / \partial t=-k_{1}\left(E_{0}-X\right) S+k_{-1} X+D \nabla^{2} S \\
& \partial X / \partial t=k_{1}\left(E_{0}-X\right) S-\left(k_{-1}+k_{2}\right) X
\end{aligned}
$$

where, as before, we represent the concentration of each reactant by the letters specified in (24).

The Qss equations for this system are obtained by setting $\partial X / \partial t=0$ :

$$
\begin{aligned}
\frac{\partial S}{\partial t} & =-k_{1}\left(E_{0}-\frac{E_{0} S}{S+K}\right) S+k_{-1} \frac{E_{0} S}{S+K}+D \nabla^{2} S, \\
X & =E_{0} S /(S+K),
\end{aligned}
$$

where, as previously, $K=\left(k_{-1}+k_{2}\right) / k_{1}$.

Including diffusion greatly increases the complexity of the system and only in certain special cases can an analytic solution be found. For example, if $K$ is very large then, independent of the substrate concentration, $\epsilon$ is small and we would expect the QSSA to hold everywhere. In many enzyme reactions it is common for $K$ to be large (Stryer 1981) because $k_{-1}$ is much larger than $k_{1}$. Assuming that this is the case, we neglect the $k_{1} E_{0} S^{2} /(S+K)$ term in (27), as it is much smaller than the other two reaction terms on the right-hand side of the equation, and we assume that $S+K \approx K$. This reduces the nonlinear equation (27) to the linear equation

$$
\frac{\partial S}{\partial t}=-\frac{k_{1} k_{2}}{k_{-1}+k_{2}} E_{0} S+D \nabla^{2} S
$$

which, on a radially symmetric, two-dimensional domain, with initial condition (23) has solution (see, for example, Crank 1956)

$$
S=\frac{1}{4 \pi D t} \exp \left[-\frac{k_{1} k_{2}}{k_{-1}+k_{2}} E_{0} t-\frac{r^{2}}{4 D t}\right] .
$$

In figure 1 we compare this solution to numerical solution of the full system (25) and (26) for various times and points in space. The analytic solution is clearly in very good agreement with the numerical solution confirming that the QSsA is valid for large $K$. 
(a)
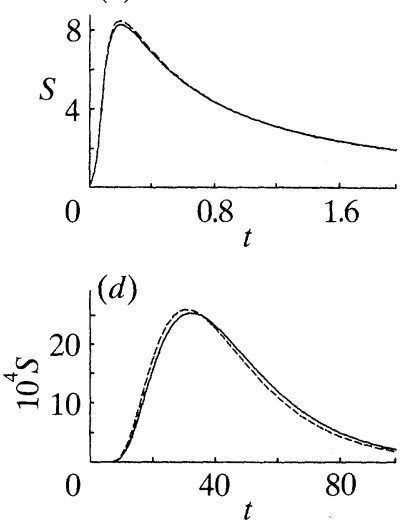

(b)
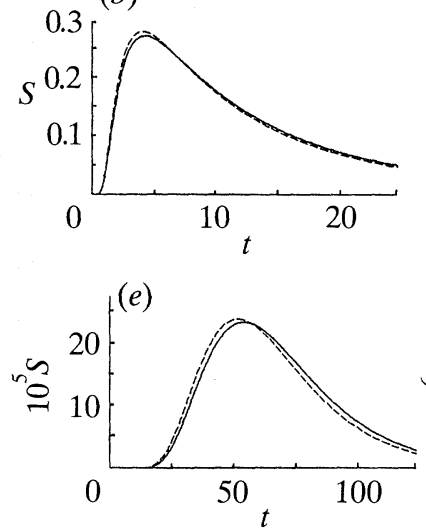

(c)
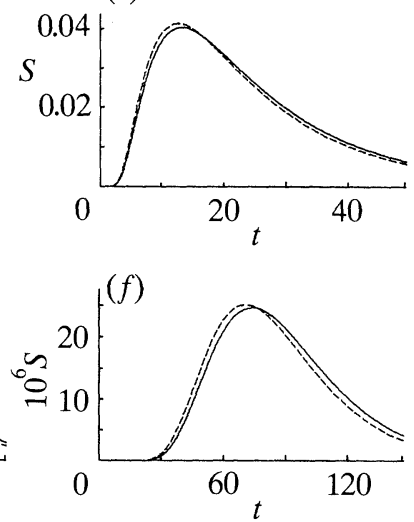

Figure 1. Comparison of the analytic solution (dashed line, equation (30)) for the Michaelis-Menten reaction with the numerical solution for the full problem (solid line, equations (25) and (26)) in the radially symmetric case for a two-dimensional domain. Plots show the time evolution of substrate concentration, $S(r, t)$, at various distances $r$ from the origin. $(a) r=0.2$. (b) $r=1.0$. (c) $r=2.0$. (d) $r=4.0$. (e) $r=6.0$. (f) $r=8.0$. Parameters: $k_{1}=1.0, k_{-1}=20.0, k_{2}=1.0, k_{3}=1.0, E_{0}=1.0$, $S_{0}=4.0$. For the numerical solution, the initial condition (23) is approximated by a tent function. Therefore the error between the two solutions is composed of the error induced by approximating the initial condition and that induced by using the QSSA.

The corresponding QsS equations for the suicide substrate system are

$$
\begin{gathered}
\frac{\partial S}{\partial t}=-\frac{k_{2} S\left(E_{0}-E_{\mathrm{i}}\right)}{S+k_{2} S /\left(k_{3}+k_{4}\right)+K}+D \nabla^{2} S, \\
\frac{\partial E_{\mathrm{i}}}{\partial t}=\frac{k_{2} k_{4}}{k_{3}+k_{4}}\left[\frac{S\left(E_{0}-E_{\mathrm{i}}\right)}{S+k_{2} S /\left(k_{3}+k_{4}\right)+K}\right],
\end{gathered}
$$

with initial conditions

$$
S(r, 0)=S_{0} \delta(r), \quad E_{\mathrm{i}}(r, 0)=0 .
$$

These equations do not admit an explicit analytic solution but numerical simulations show that they are in good agreement with the numerical solutions of the full system (11)-(14) thus illustrating, again, that the QSSA is valid for large $K$.

If $K=O\left(E_{0}\right)$, then for a point distance $z$ away from the origin, $\epsilon(z)$ will be $O(1)$ initially (because $S_{0}(z)$, the initial substrate concentration at $z$ is zero) and the system will not be in QSS at $z$. As the substrate diffuses away from the origin the value of $S$ at $z$, namely $S(z)$, will rise and in time $\epsilon(z)$ may indeed become small and the reaction will move into Qss. As the reaction proceeds further, substrate will be used up and will also diffuse away from $z$ so $S(z)$ will decrease. However, during the reaction at $z$, some of the enzyme will have been inactivated so that $E$ has also decreased. Thus it is still possible for $\epsilon(z)$ to be small. Hence we expect that there will be a growing disc, centre the origin, in which the Qss holds. Note that if we were dealing with a reaction in which the enzyme were not inactivated - for example, in the Michaelis-Menten reaction - then $\epsilon(z)$ would become $O(1)$ again. Therefore we should first see a circular disc in which the QSs holds move out from the origin followed by a second, concentric, disc in which the expression defined by $\epsilon$ becomes $O(1)$ and the Qss no longer holds. The result is a kind of travelling ring in which the QSs holds. 

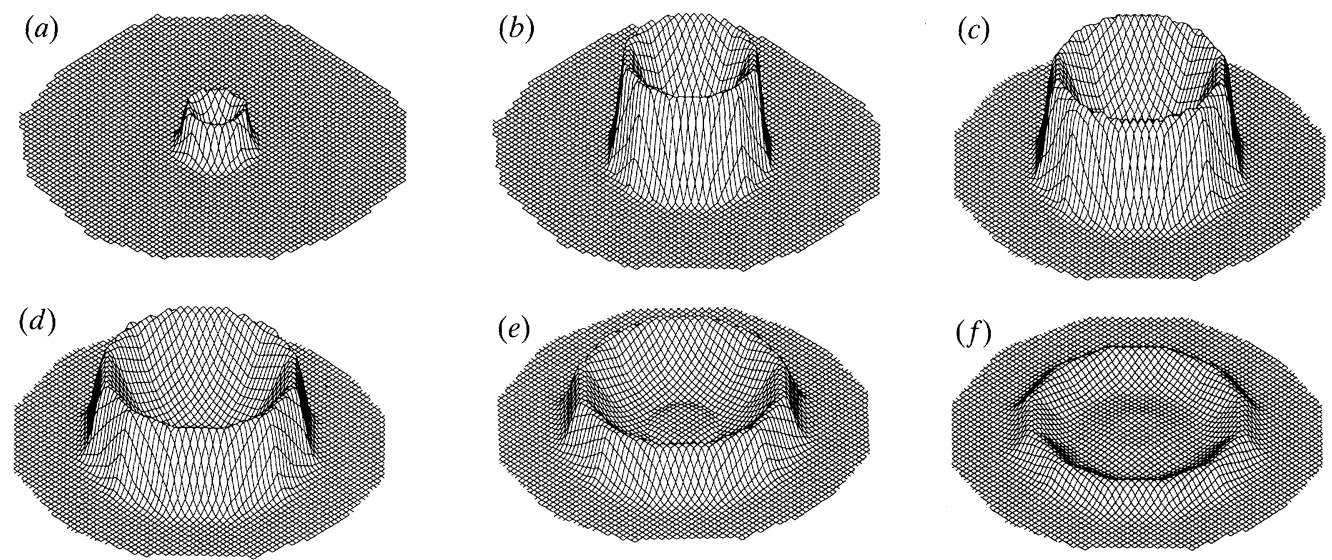

Figure 2. Zone of validity of the QSSA for the suicide substrate system. The difference between the substrate concentration obtained by solving the QSS equations (31) and (32) and that given by solving the full system (11)-(14), as a percentage of their average, for the radially symmetric case in two dimensions. Flat regions correspond to points in the plane where the QSSA is valid. All solutions are computed numerically for different times, $t$. $(a) t=0.1$, maximum difference $=20 \%$. (b) $t=0.5$, maximum difference $=47 \%$. (c) $t=1.0$, maximum difference $=48 \%$. (d) $t=1.5$, maximum difference $=36 \%$. (e) $t=2.0$, maximum difference $=22 \%$. (f) $t=2.5$, maximum difference $=10 \%$. Parameters : $k_{1}=1.0, k_{-1}=1.0, k_{2}=1.0, k_{3}=1.0, k_{4}=1.0, E_{0}=1.0, S_{0}=40.0$.
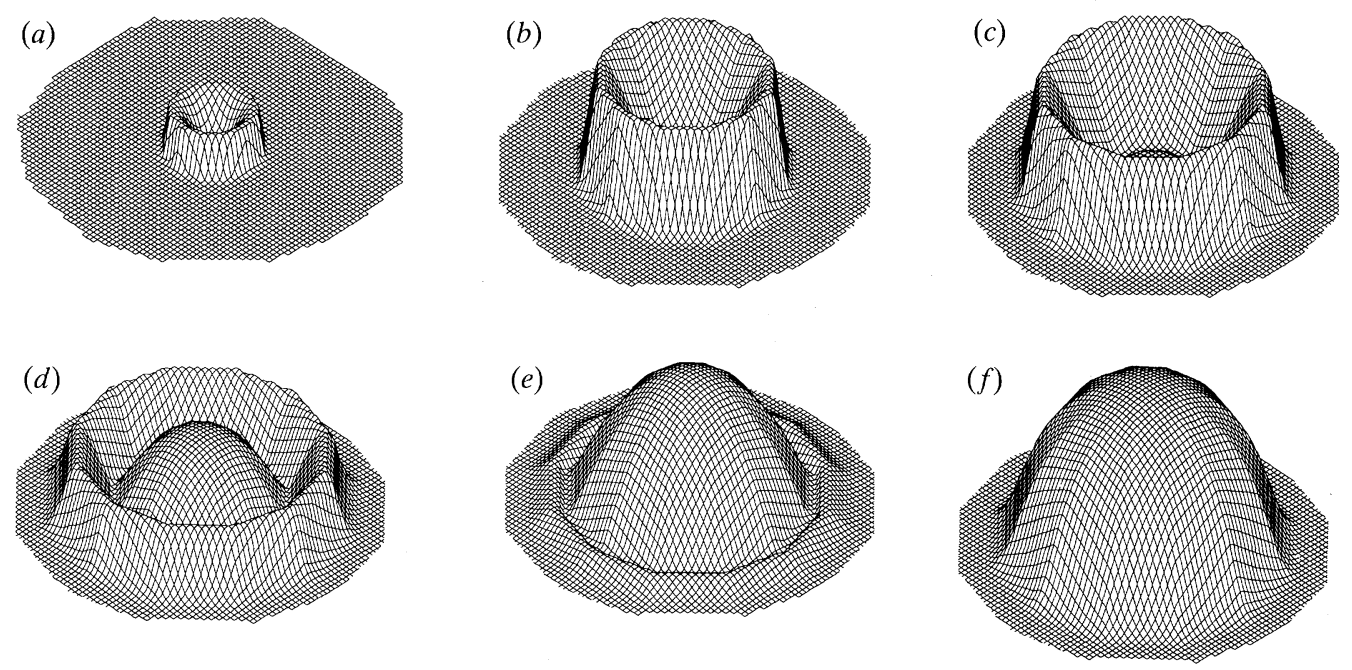

Figure 3. Zone of validity of the QSSA for the Michaelis-Menten system. The difference between the substrate concentration obtained by solving the Qss equations (27) and (28) and that given by solving the full system (25) and (26), as a percentage of their average, for the radially symmetric case in two dimensions. Flat regions correspond to points in the plane where the QSSA is valid. All solutions are computed numerically for different times, $t$. (a) $t=0.1$, maximum difference $=19 \%$. (b) $t=0.5$, maximum difference $=47 \%$. (c) $t=1.0$, maximum difference $=46 \%$. (d) $t=1.5$, maximum difference $=35 \%$. (e) $t=2.0$, maximum difference $=60 \%$. (f) $t=2.5$, maximum difference $=76 \%$. Parameters : $k_{1}=1.0, k_{-1}=1.0, k_{2}=1.0, k_{3}=1.0, E_{0}=1.0, S_{0}=40.0$.

In figures 2 and 3 we plot the difference between the solution to the Qss equations and the solution to the corresponding full system for the suicide substrate and the Michaelis-Menten systems respectively. The disc in which the Qssa holds for 
the suicide substrate system and the corresponding ring in the case of the Michaelis-Menten reaction are clearly seen.

\section{Discussion}

We have considered enzyme-suicide-substrate and Michaelis-Menten kinetics in which the substrate is diffusing. Previous analysis for the non-diffusing substrate case shows that the size of the ratio $E_{0} /\left(S_{0}+K\right)$ determines the parameter domain in which the QSSA is valid. We have extended this idea to determine the domain in space and time in which the QSSA is valid for a diffusing substrate. In particular we have shown that the QSSA is valid in an expanding disc for the suicide substrate case but only in a ring for the Michaelis-Menten reaction.

Including diffusion increases considerably the complexity of the problem and, only in special cases, is it amenable to an analytical treatment. We presented one such case here and obtained an analytic solution valid only in the QSs phase. To obtain a uniformly valid solution one much carry out a subtle analysis of the pre-steady-state phase of the reaction and match to the outer QSS phase: this will be reported elsewhere.

This material is based upon work supported under a National Science Foundation Graduate Fellowship (M.A.B.). M.A.B. acknowledges the Universities of the United Kingdom Overseas Research Scheme, and J.D.M. acknowledges the US National Science Foundation (Grant DMS9003339).

\section{References}

Burke, M. A., Maini, P. K. \& Murray, J. D. 1990 On the kinetics of suicide substrates. Biophys. Chem. 37, 81-90.

Crank, J. 1956 The mathematics of diffusion. Clarendon Press.

Frenzen, C. L. \& Maini, P. K. 1988 Enzyme kinetics for a two-step enzymic reaction with comparable initial enzyme-substrate ratios. J. math. Biol. 26, 689-703.

Laidler, K. J. \& Bunting, P. S. 1977 The chemical kinetics of enzyme action. Oxford: Clarendon Press.

Lin, C. C. \& Segel, L. A. 1974 Mathematics applied to deterministic problems in the natural sciences. New York: Macmillan.

Michaelis, L. \& Menten, M. L. 1913 Die kinetik der invertinwirkung. Biochem. Z. 49, 333-369.

Murray, J. D. 1989 Mathematical biology. Heidelberg: Springer-Verlag.

Rando, R. R. 1977 Mechanism-based irreversible enzyme inhibitors. Methods Enzymol. 46, 28-41.

Segel, L. A. \& Slemrod, M. 1989 The quasi-steady-state assumption : a case study in perturbation. SIAM Rev. 31, 446-477.

Seiler, N., Jung, M. J. \& Koch-Weser, J. (eds) 1978 Enzyme-activated irreversible inhibitors. Oxford: Elsevier/North-Holland Biomedical Press.

Sols, A. \& Marco, R. 1970 Concentrations of metabolites and binding sites. Implications in metabolic regulation. In Current topics in cellular regulation (ed. B. Horecker \& E. Stadman), Vol. 2, pp. 227-273. New York: Academic Press.

Stryer, L. 1981 Biochemistry, 2nd edn. New York: W. H. Freeman.

Tatsunami, S., Yago, N. \& Hosoe, M. 1981 Kinetics of suicide substrates : steady-state treatments and computer-aided exact solutions. Biochim. Biophys. Acta 662, 226-235.

Waley, S. G. 1980 Kinetics of suicide substrates. Biochem. J. 185, 771-773.

Walsh, C., Cromartie, T., Marcotte, P. \& Spencer, R. 1978 Suicide substrates for flavoprotein enzymes. Meth. Enzymol. 53, 437-448.

Walsh, C. T. 1984 Suicide substrates, mechanism-based enzyme inactivators: Recent developments. A. Rev. Biochem. 53, 493-535. 